\title{
Source-specific light absorption by carbonaceous components in the complex aerosol matrix from yearly filter-based measurements
}

Vaios Moschos ${ }^{1}$, Martin Gysel-Beer ${ }^{1}$, Robin L. Modini ${ }^{1}$, Joel C. Corbin ${ }^{2}$, Dario Massabò ${ }^{3}$, Camilla Costa ${ }^{3}$, Silvia G. Danelli ${ }^{3}$, Athanasia Vlachou ${ }^{1}$, Kaspar R. Daellenbach ${ }^{1}$, Sönke Szidat ${ }^{4}$, Paolo Prati ${ }^{3}$, André S. H. Prévôt $^{1}$, Urs Baltensperger ${ }^{1}$, and Imad El Haddad ${ }^{1}$

${ }^{1}$ Laboratory of Atmospheric Chemistry, Paul Scherrer Institute, Villigen-PSI, CH-5232, Switzerland

${ }^{2}$ Metrology Research Centre, National Research Council Canada, Ottawa, ON K1A 0R6, Canada

${ }^{3}$ Department of Physics/(Industrial) Chemistry \& INFN, University of Genoa, Genova, I-16146, Italy

${ }^{4}$ Department of Chemistry and Biochemistry / Oeschger Centre for Climate Change Research, University of Bern, Bern, CH-3012, Switzerland

Correspondence to: Imad El Haddad (imad.el-haddad@psi.ch)

\section{SUPPORTING TEXT}

\section{S1. Filter sampling}

In total, $245 \mathrm{PM}_{10}$ filters from Magadino (Jan '13 - Sep '14) and Zurich (Jan - Dec '13), and 65 PM 2.5 filters from Magadino (Jan - Sep '14), were collected over $24 \mathrm{~h}$ every $4^{\text {th }}$ day, with a total sampled air volume of $720 \mathrm{~m}^{3}$ per filter. Zurich-Kaserne is an urban background site, located in a courtyard in the city center of Zurich (410 m.a.s.l.). The location is surrounded by roads with rather low traffic and is not affected by major emissions from industries. Magadino-Cadenazzo is a rural background air quality monitoring station located in Southern Switzerland, south of the Alps in the Magadino plane close to the Lago Maggiore, about two kilometers outside the small village of Cadenazzo. The site is surrounded by agricultural land and forests and is exposed to wood smoke from residential heating during winter. Biomass burning at both sites might be associated with domestic heating during wintertime and with open fires, agricultural waste disposal, waste burning etc., during the other seasons.

\section{S2. Measurements of aerosol size distribution and morphology}

Particle (number) size distributions were measured in Zurich during 2013 using a Scanning Mobility Particle Sizer (SMPS) at 54 particle size bins between 10 to $487 \mathrm{~nm}$. Data were collected continuously throughout the year with a time resolution of 10 min and averaged over $24 \mathrm{~h}$ every $4^{\text {th }}$ day in order to have the same time resolution as the respective filter samples (sample number $=88,3$ daily samples were excluded due instrumental issues). Volume size distributions were derived assuming spherical particles and the contribution of the different components to the mass size distributions were obtained by assuming identical particle density for all components (see Figure S7).

A field-emission scanning electron microscope (Zeiss Supra $40 \mathrm{VP}$ ) coupled with an energy-dispersive X-ray spectrometer (FESEM/EDS) was used to observe the general morphology and deposition characteristics on the fiber filters, as well as the elemental composition of individual (non-)carbonaceous particles. The FE-SEM scanned different filter areas at magnifications up to 100000x with accelerating voltage of 5-10-20 kV, beam current value of $200 \mathrm{nA}$ and work distance of $3.5 \mathrm{~mm}$ [secondary electrons (SE) inlens detector]. An energy-dispersive X-ray spectrometer (EDS, Oxford Link with a Pentafet Si(Li) detector) was used to detect elements. We chose two representative winter samples, one from each site during winter with the highest BBOA contribution to OA mass.

\section{S3. Optical measurements}

\section{S3.1: Total aerosol absorption}

For both Zurich and Magadino, the total aerosol attenuation of daily samples $j, b_{A T N, A E 33, j, i}$, was measured online at sevenwavelengths, $(370,470,520,590,660,880$ and $950 \mathrm{~nm})$ using a dual-spot Aethalometer (AE33 model, Magee Scientific) at a time resolution of $10 \mathrm{~min}$. Firmware output of loading compensation was used. In the Aethalometer, light is transmitted through a particleladen filter $(I)$ and the effective attenuation coefficient is measured by comparing the transmission to that of a clean filter $\left(I_{0}\right)$. Daily averaged $b_{A T N, A E 33, j}(\lambda)$ were calculated, corresponding to the offline filter samples. For selected set of 27 daily offline samples (22 mm circular punches), aerosol absorption $\left(b_{a b s, M W A A, j}(\lambda)\right.$, in $\left.\mathrm{Mm}^{-1}\right)$ was determined at five wavelengths using the multi-wavelength absorption analyzer (MWAA) (Massabò et al., 2013). The MWAA measures the transmission and backward scattering at two fixed angles. Measurements were performed at $375,407,532,635$ and $850 \mathrm{~nm}$, and power law fitting $\left(\mathrm{R}^{2}>0.97\right)$ was applied to interpolate the data to compare with the AE33 attenuation measurements. From these comparisons, wavelength-specific AE33 calibration coefficients, $C(\lambda)$, were derived using Eq. (S1): 
$C(\lambda)=\frac{b_{A T N, A E 33, j}(\lambda)}{b_{a b s, M W A A, j}(\lambda)}$

The mean $C(\lambda)$ values were then used to obtain from AE33 data aerosol absorption coefficients, $b_{a b s, t o t a l, j}(\lambda)$, for the entire sample set (Eq. (S2)):

$b_{\text {abs, total }, j}(\lambda)=\frac{b_{A T N, A E 33, j}(\lambda)}{C(\lambda)}$

The 1-SD of $C(\lambda)$ variability was propagated through Eq. (S2) to obtain the respective 1 SD uncertainty on $b_{a b s, t o t a l, j}(\lambda)$.

Similar to Corbin et al. (2019), MWAA measurements were carried out also upon extraction (using small petri dishes) of 4 Magadino 2014 and 5 Zurich 2013 PM$_{2.5}$ filters in $5 \mathrm{~mL}$ water and then in $5 \mathrm{~mL}$ methanol $(1 \mathrm{~h}, 70 \mathrm{rpm})$. Results are blank-subtracted in all cases. The repeats of original or water-washed punch measurements showed very good reproducibility in the AAE values, but not always accurate in terms of absolute absorbance. This is likely due to mechanical removal of insoluble particles, as we could confirm through SEM analysis of evaporated aerosol extracts (Fig. S13). Therefore, here we used only the AAE values as a measure of the wavelength dependence of the remaining aerosol, under the assumption that particle mechanical removal with extraction is not selective to certain insoluble aerosol.

\section{S3.2: Absorption of aerosol extracts}

The experimental setup and detailed analytical protocol for absorption measurement in extracts were described in our earlier publication (see SI of Moschos et al., 2018). Briefly, attenuation spectra of aerosol extracts in ultrapure water or methanol were measured from 280 to $600 \mathrm{~nm}$ using a UV-visible spectrophotometer (Ocean Optics) coupled to long-path detection cell (length $l=$ $50 \mathrm{~cm}$ ). The instrument records the light attenuation in solution, $A T N_{\text {sol }}^{10}(\lambda)$, for a sample $j$ as a function of wavelength, as the decadic logarithm of the ratio of signal intensities of the reference (solvent, $I_{0}$ ) and the sample $(I)$, both corrected for background signals with the light source off. From $A T N_{s o l}^{10}(\lambda)$, the absorption coefficient in solution (in $\left.\operatorname{Mm}^{-1}\right), A=A b s_{U V-v i s, j}(\lambda)$, can be quantified (Hecobian et al., 2010) based on the Beer-Lambert law (Eq. (S3)), assuming that additional light scattering by the solute can be neglected upon dissolution:

$A_{j}(\lambda)=A b s_{U V-v i s, j}(\lambda)=\frac{A T N_{\text {sol }_{j}}^{10}(\lambda)}{l} \cdot \ln 10 \cdot\left(\frac{V_{\text {sol }}}{V_{\text {air }}}\right)_{j}$

where $V_{\text {air }}$ and $V_{\text {sol }}$ are the volumes of air sampled through the filter (punch) and solvent used during the extraction (both in $\mathrm{m}^{3}$ ), respectively. In this work, when the solvent was methanol, the extract volume was 3 or $6 \mathrm{~mL}$ and PTFE syringe filters of same dimensions $(0.45 \mu \mathrm{m})$ were used $(0.22 \mathrm{vs} 0.45 \mu \mathrm{m}$ filtering did not have any noticeable effect on the recorded spectra).

\section{S4. UV/Vis-PMF applications}

We performed an exploratory analysis (Table S1) to determine which AMS/PMF factors from each solution drive the measured absorbance in methanol. This analysis was based on the correlation between factor mass and absorbance, for the full dataset of each factor solution, and for selected time points where each factor dominates in terms of mass compared to other absorbing fractions. The outcome of this analysis pointed to the inclusion of all OA factors, except for SCOA and PBOA that contribute significantly to the coarse mode $\left(\mathrm{PM}_{10}-\mathrm{PM}_{2.5}\right)$ mass, in the UV/Vis-PMF model. Also, the absorbance of the coarse fraction in Magadino during 2014 contributed significantly less to $\mathrm{PM}_{10}$ absorbance than its respective contribution to $\mathrm{PM}_{10}$ mass (Fig. S8). HOA, COA, fOOA and SOOA were combined into a single (for each AMS/PMF solution) "Other OA" factor before UV/Vis-PMF analysis, because initial model runs showed their individual contributions to absorbance to be consistently lower than those of BBOA and WOOA. It was also confirmed, by considering all OA factors in the model, that the scaled residuals were not further reduced by including SCOA and PBOA (Fig. S9), which further demonstrated that these fractions did not contribute significantly to absorbance of the $\mathrm{PM}_{10}$ aerosol fraction. UV/Vis-PMF (Moschos et al., 2018) was then constrained using three factors (BBOA, WOOA, Other OA) and was applied to both methanol-soluble $\left(A_{\mathrm{MeOH}, j}(\lambda)\right)$ and water-insoluble $\left(A_{\mathrm{MeOH}, j}(\lambda)-A_{\mathrm{H} 2 \mathrm{O}, \mathrm{j}}(\lambda)\right)$ absorbance matrices, where $A_{H 2 O, j}(\lambda)$ was corrected for the water/methanol solvent effect (Fig. S5). The application of UV/Vis-PMF on the water-insoluble absorbance data showed that, unlike BBOA, WOOA and Other OA did not explain a quantifiable portion of the absorbance (too low or noisy contributions). For example, for case (g) mentioned in this section, water-insoluble BBOA could explain the totality of the input water-insoluble absorbance at $370 \mathrm{~nm}$. For the calculation of $k(\lambda)$ for methanol-soluble BBOA and water-insoluble (but methanol-soluble) BBOA, we multiplied the total AMS/PMF BBOA mass with 0.93 to account for the average extraction efficiency of BBOA in methanol (Fig. S6). Also, the estimated solubility of WOOA and BBOA in water was up to $90 \%$ and $66 \%$, respectively, based on the calculated recoveries by AMS/PMF analysis (Daellenbach et al., 2017; Vlachou et al., 2018). WOOA can thus be considered fully water-soluble. We examined the uncertainties in $k(\lambda)$ (or $M A E(\lambda)$ ) by varying the sample size of the input matrices, using the respective factor mass concentrations from the offline AMS/PMF Solutions 1 and 2. Specifically, the spatial coverage and temporal coverage or resolution were reduced compared to the full dataset runs for each PM $\mathrm{PM}_{10} \mathrm{AM} / \mathrm{PMF}$ factor solution, as follows (the retrieved factor-specific $k$ at $370 \mathrm{~nm}$ from the different sensitivity runs is shown in Table S2):

a) Solution 2: full dataset; Magadino and Zurich 2013 (182 time points)

b) Solution 2: Magadino 2013 alone (91 time points)

c) Solution 2: Zurich 2013 alone (91 time points)

d) Solution 2: first two filters per month; Magadino and Zurich 2013 (48 time points) 
e) Solution 2: last two filters per month; Magadino and Zurich 2013 (48 time points)

f) Solution 1: full dataset; Magadino 2013/14 (154 time points)

g) Solution 1: Magadino 2013/14 winter (64 time points)

h) Solution 1: Magadino 2013/14 summer (90 time points)

i) Solution 1: first two filters per month; Magadino 2013/14 (42 time points)

j) Solution 1: last two filters per month; Magadino 2013/14 (42 time points)

\section{S5. Calculation of MAC/MAE $370 \mathrm{~nm}$}

The factor contributions to particulate $\mathrm{BrC}$ absorption were obtained by considering the following cases for the source-specific $\mathrm{BrC}$ particle size and mixing states with other NR-PM (OOA = sum of the different OOA factors):

Case 1; BBOA in the smaller size range $(120 \mathrm{~nm})$, externally mixed with inorganics; OOA in the larger size range (200-400 $\mathrm{nm})$, externally mixed with inorganics.

Case 2; BBOA in the larger size range (200-400 nm), externally mixed with inorganics; OOA in the larger size range (200-400 $\mathrm{nm})$, externally mixed with inorganics.

Case 3; BBOA in the larger size range (200-400 nm), externally mixed with inorganics; OOA in the larger size range (200-400 $\mathrm{nm})$, internally mixed with inorganics.

Case 4; BBOA in the smaller size range $(120 \mathrm{~nm})$, externally mixed with inorganics; OOA in the larger size range (200-400 $\mathrm{nm})$, internally mixed with inorganics.

Case 5; BBOA+OOA+inorganics, all internally mixed in the larger size range (200-400 nm).

Case 6; BBOA in the smaller size range $(120 \mathrm{~nm})$, externally mixed with inorganics; OOAs externally mixed from each other in the larger size range (200-400 nm), externally mixed with inorganics.

Case 7; BBOA in the larger size range (200-400 nm), externally mixed with inorganics; OOAs externally mixed from each other in the larger size range $(200-400 \mathrm{~nm})$, externally mixed with inorganics.

We considered an OOA particle size of 200-400 nm ("larger size range") and a lower limit of $\sim 120$ nm for primary (non-OOA) BrC, based on available SMPS data for Zurich (Fig. S7). Fresh BBOA is known in certain cases to have smaller diameter, e.g., from SP2 measurements in Paris (Laborde et al., 2013). Therefore, we have considered the above cases where BBOA occurs in smaller sizes. HOA and COA were treated as externally mixed small size particles except for "case 5". Furthermore, past size distributions from Zurich (Wolf et al., 2017) indicate that (W)OOA most likely resides in the accumulation mode. PBOA and SCOA were not considered in these cases, because these fractions reside mainly in the coarse mode and thus are not expected to be internally-mixed with other aerosol fractions.

For each case, we obtained the effective $k, k_{\text {mix }}$, of each particle type, $p$ (Eq. (S4)), by using the median wavelength-dependent $k(\lambda)$ of methanol-soluble WOOA, BBOA and Other OA estimated by UV/Vis-PMF, multiplied with the mass fraction $(f)$ of each aerosol component $X$ in the different particle mixtures (volume mixing rule):

$k_{\text {mix }, j}(\lambda)=\sum_{X}\left[\overline{\bar{k}}_{X}(\lambda) \cdot f\left\{[X]_{p, j}\right\}\right]$

We converted the resulting $k_{\text {mix }, j}(\lambda)$ to mass absorption cross-sections (MAC) at four AE33 wavelengths $(370 \mathrm{~nm}, 470 \mathrm{~nm}, 520$ $\mathrm{nm}, 590 \mathrm{~nm}$ ) with Mie code programmed in the software package Igor Pro (WaveMetrics). The resulting mass absorption crosssections of each particle type, $\operatorname{MAC}_{j}(p, \lambda)$, were multiplied with the total mass concentrations of its constituents to obtain the respective absorption coefficients, $b_{a b s_{M i e, j}}^{B r C-p}(\lambda)$, at the different AE33 wavelengths, using Eq. (S5):

$b_{a b s_{\text {Mie,j }}}^{\operatorname{BrC}-p}(\lambda)=[X]_{p, j} \cdot \operatorname{MAC}_{j}(p, \lambda)$

The retrieved $b_{a b s_{M i e, j}}^{B r C-p}(\lambda)$ values for each particle type were summed to obtain the total absorption coefficient from all BrCcontaining particles, $b_{a b s, B r C-M i e, j}(\lambda)$ for each sample and each of the seven cases, using Eq. (S6):

$b_{a b s, B r C-M i e, j}(\lambda)=\sum_{p} b_{a b s, B r C-M i e, j}(p, \lambda)$

The factor-specific and total $\mathrm{BrC}$ MAC/MAE values at $370 \mathrm{~nm}$ from the seven distinct mixing state cases are summarized in Table $\mathrm{S} 3$. The calculations for total MeOH-BrC (last column) were based on the following notation: $b_{a b s, B r C-M i e}=(M A C / M A E)_{N R-P M} *$ $M A E_{\text {totaloA }} *[O A]$, where $M A E_{N R-P M} *[N R-P M]=M A E_{\text {totaloA }} *[O A]$. The MAC/MAE uncertainty corresponds to the full range between the min. and max. total $\mathrm{BrC}$ absorption values from the 7 cases. 


\section{SUPPORTING TABLES \& FIGURES}

Table S1. Exploratory analysis for AMS/PMF factor selection in the UV/Vis-PMF model. The individual factors eventually considered to constrain the model were based on specific correlation criteria; no fully transparent OA fractions were included in the model, i.e., $\mathrm{R}^{2}$ between factor mass time series and bulk methanol solution absorbance ( $\mathrm{PM}_{10}$ or coarse) time series $<0.4$, for selected time points where each $\mathrm{OA}$ factor ( $\mathrm{PM}_{10}$ or coarse) mass dominates compared to that of other absorbing factors (coarse data existed only for data from 2014, so the correlations with the coarse fraction could be tested only for Solution 1; only positive values for both the coarse fraction mass and absorbance were used). Note that the Pearson's $r$ was positive in all cases except for the coarse PBOA (respective $\mathrm{R}^{2}$ value indicated with an asterisk).

\begin{tabular}{|c|c|c|c|}
\hline Factor & Mass ratio criterion & $\begin{array}{l}\text { Number of } \\
\text { time points }\end{array}$ & $\begin{array}{l}\mathbf{R}^{2} \text {; Factor mass vs bulk } \\
\text { absorbance time series }\end{array}$ \\
\hline \multicolumn{4}{|c|}{ AMS/PMF «Solution 2» } \\
\hline \multirow{4}{*}{ BBOA } & (Block) & 182 & 0.92 \\
\hline & BBOA:WOOA>3 & 27 & 0.98 \\
\hline & WOOA:BBOA>3 & 42 & 0.75 \\
\hline & SOOA:(BBOA+WOOA)>3 & 32 & 0.36 \\
\hline \multirow{4}{*}{ WOOA } & (Block) & 182 & 0.36 \\
\hline & WOOA:BBOA>3 & 42 & 0.83 \\
\hline & BBOA:WOOA>3 & 27 & 0.80 \\
\hline & SOOA:(BBOA+WOOA)>3 & 32 & 0.38 \\
\hline \multirow{2}{*}{ SOOA } & (Block) & 182 & 0.06 \\
\hline & SOOA:(BBOA+WOOA)>3 & 32 & 0.56 \\
\hline \multirow{2}{*}{ HOA } & (Block) & 182 & 0.17 \\
\hline & HOA:(BBOA+WOOA)>1 & 29 & 0.71 \\
\hline \multirow{2}{*}{ COA } & (Block) & 182 & 0.12 \\
\hline & $\mathrm{COA}:(\mathrm{BBOA}+\mathrm{WOOA})>1$ & 25 & 0.78 \\
\hline \multirow{2}{*}{ SCOA } & (Block) & 182 & 0.17 \\
\hline & SCOA:(BBOA+WOOA)>1 & 39 & 0.37 \\
\hline \multicolumn{4}{|c|}{ AMS/PMF «Solution 1» } \\
\hline \multirow{4}{*}{ BBOA } & (Block) & 154 & 0.90 \\
\hline & BBOA:WOOA>3 & 59 & 0.93 \\
\hline & WOOA:BBOA>3 & 27 & 0.79 \\
\hline & SOOA:(BBOA+WOOA)>3 & 55 & 0.24 \\
\hline \multirow{4}{*}{ WOOA } & (Block) & 154 & 0.21 \\
\hline & WOOA:BBOA>3 & 27 & 0.96 \\
\hline & BBOA:WOOA>3 & 59 & 0.30 \\
\hline & SOOA:(BBOA+WOOA)>3 & 55 & 0.44 \\
\hline \multirow{2}{*}{ SOOA } & (Block) & 154 & 0.25 \\
\hline & SOOA:(BBOA+WOOA)>3 & 55 & 0.54 \\
\hline \multirow{2}{*}{ HOA } & (Block) & 154 & 0.22 \\
\hline & HOA:(BBOA+WOOA)>1 & 25 & 0.45 \\
\hline \multirow{2}{*}{ fOOA } & (Block) & 154 & 0.05 \\
\hline & fOOA:(BBOA+WOOA)>2 & 37 & 0.42 \\
\hline \multirow{2}{*}{ SCOA } & (Block) & 154 & 0.09 \\
\hline & SCOA:(BBOA+WOOA)>1 & 21 & 0.00 \\
\hline \multirow{2}{*}{ PBOA } & (Block) & 154 & 0.06 \\
\hline & PBOA:(BBOA+WOOA)>4 & 34 & 0.17 \\
\hline SCOA (coarse) & (Block) & 154 & 0.11 \\
\hline PBOA (coarse) & (Block) & 154 & $0.30 *$ \\
\hline
\end{tabular}


Table S2. UV/Vis-PMF-based $k$ of the methanol-soluble BrC factors at $370 \mathrm{~nm}$ from different sensitivity runs.

\begin{tabular}{|r|c|c|c|c|}
\hline k $_{\text {370nm }}$ & BBOA & WOOA & Other OA & WINS-BBOA \\
\hline $\mathrm{a}$ & 0.067 & 0.025 & 0.0064 & 0.075 \\
\hline $\mathrm{b}$ & 0.070 & 0.024 & 0.0010 & 0.082 \\
\hline $\mathrm{c}$ & 0.078 & 0.030 & 0.0057 & 0.127 \\
\hline $\mathrm{d}$ & 0.067 & 0.030 & 0.0057 & 0.094 \\
\hline $\mathrm{e}$ & 0.069 & 0.025 & 0.0077 & 0.083 \\
\hline $\mathrm{f}$ & 0.046 & 0.038 & 0.0043 & 0.039 \\
\hline $\mathrm{g}$ & 0.046 & 0.041 & 0.0046 & 0.042 \\
\hline $\mathrm{h}$ & 0.058 & 0.041 & 0.0026 & 0.063 \\
\hline $\mathrm{i}$ & 0.045 & 0.031 & 0.0079 & 0.045 \\
\hline $\mathrm{j}$ & 0.046 & 0.025 & 0.0080 & 0.044 \\
\hline Q1 & 0.046 & 0.025 & 0.0044 & 0.044 \\
\hline median & 0.062 & 0.030 & 0.0057 & 0.069 \\
\hline Q3 & 0.068 & 0.036 & 0.0074 & 0.083 \\
\hline
\end{tabular}

Table S3. Factor-specific and total BrC MAC/MAE at $370 \mathrm{~nm}$ obtained for seven distinct mixing state cases through Eq. (S4). The " \pm " sign indicates the day-to-day variability for all $245 \mathrm{PM}_{10}$ filter samples from Magadino and Zurich. The full-dataset MAC/MAE for HOA, COA, SOOA and fOOA was calculated using the UV/Vis-PMF output median $k$ of the combined "Other OA" factor (Fig. 2) and each factor mass from the respective AMS/PMF factor solution. Note that for "case 5" (fully internally mixed BBOA+OOAs+inorganics), the conversion factor is identical for all absorbing AMS/PMF source components. The min and max (from the 7 cases) MAC/MAE were used to convert the UV/Vis-PMF-based median $k$ (Fig. 2) to factor-specific particulate MAC values [min, max] in the last row, considering the MAC/MAE uncertainty.

\begin{tabular}{|c|c|c|c|c|c|c|c|c|}
\hline $\begin{array}{c}\text { MAC:MAE } \\
\text { @ } 370 \mathrm{~nm}\end{array}$ & BBOA & WOOA & $\begin{array}{c}\text { Other OA } \\
\text { (combined) }\end{array}$ & HOA & COA & SOOA & fOOA & $\begin{array}{c}\text { Total } \\
\text { MeOH-BrC }\end{array}$ \\
\hline Case 1 & 1.05 & $1.66 \pm 0.04$ & $1.48 \pm 0.17$ & 1.09 & 1.09 & $1.66 \pm 0.04$ & $1.68 \pm 0.03$ & $1.33 \pm 0.16$ \\
\hline Case 2 & 1.35 & $1.66 \pm 0.04$ & $1.48 \pm 0.17$ & 1.09 & 1.09 & $1.66 \pm 0.04$ & $1.68 \pm 0.03$ & $1.48 \pm 0.08$ \\
\hline Case 3 & 1.35 & $1.71 \pm 0.02$ & $1.51 \pm 0.17$ & 1.09 & 1.09 & $1.71 \pm 0.02$ & $1.71 \pm 0.02$ & $1.50 \pm 0.09$ \\
\hline Case 4 & 1.05 & $1.71 \pm 0.02$ & $1.51 \pm 0.17$ & 1.09 & 1.09 & $1.71 \pm 0.02$ & $1.71 \pm 0.02$ & $1.35 \pm 0.17$ \\
\hline Case 5 & \multicolumn{8}{|c|}{$1.60 \pm 0.12$} \\
\hline Case 6 & 1.05 & 1.55 & $1.51 \pm 0.17$ & 1.09 & 1.09 & 1.71 & 1.71 & $1.31 \pm 0.15$ \\
\hline Case 7 & 1.35 & 1.55 & $1.51 \pm 0.17$ & 1.09 & 1.09 & 1.71 & 1.71 & $1.46 \pm 0.07$ \\
\hline$(\min +\max ) / 2$ & $1.33 \pm 0.06$ & $1.61 \pm 0.04$ & $1.55 \pm 0.09$ & $\sim 1.35$ & $\sim 1.35$ & $\sim 1.66$ & $\sim 1.66$ & $\sim 1.45$ \\
\hline $\begin{array}{c}M A C_{370 n m} \\
\left(m^{2} g^{-1}\right)\end{array}$ & {$[1.48,2.26]$} & {$[1.05,1.16]$} & $\sim 0.20$ & & & & & \\
\hline
\end{tabular}


Table S4. Different proxy results tested for the determination of bare BC MAC at $660 \mathrm{~nm}$ for three different cases, following a stepwise multiple linear regression approach. Only data points in the linear proxy (NR-PM:EC) range were considered for the linear fits. $(\mathrm{OOA}+\mathrm{BBOA}+\mathrm{m}-\mathrm{SIA}): \mathrm{EC}$ was the most consistent of the tested proxies for the three cases, in terms of the obtained MAC for bare BC, slope and Pearson's $\mathrm{r}$, indicating that OOA, BBOA and inorganics likely partitioned to coatings on EC leading to its absorption enhancement by acting as a lens.

\begin{tabular}{|l|c|c|c|}
\hline NR-PM & MAC $_{\text {bareBc }}$ (intercept) & Slope & Pearson's r \\
\hline \multicolumn{3}{|c|}{ Magadino 2013/14 winter } \\
\hline OA & $5.68 \pm 0.78$ & $0.413 \pm 0.059$ & 0.67 \\
\hline OA+m-SIA & $6.37 \pm 0.52$ & $0.236 \pm 0.025$ & 0.78 \\
\hline OOA & $7.32 \pm 0.50$ & $0.741 \pm 0.099$ & 0.70 \\
\hline OOA+m-SIA & $8.07 \pm 0.41$ & $0.231 \pm 0.028$ & 0.73 \\
\hline OOA+BBOA+HOA+m-SIA & $6.61 \pm 0.46$ & $0.240 \pm 0.020$ & 0.79 \\
\hline OOA+BBOA+m-SIA & $6.54 \pm 0.47$ & $0.261 \pm 0.026$ & 0.79 \\
\hline \multicolumn{4}{|c|}{ Magadino $2013 / 14$ summer } \\
\hline OA & $5.49 \pm 0.89$ & $0.258 \pm 0.058$ & 0.44 \\
\hline OA+m-SIA & $5.64 \pm 0.95$ & $0.194 \pm 0.048$ & 0.41 \\
\hline OOA & $6.26 \pm 0.53$ & $0.322 \pm 0.051$ & 0.56 \\
\hline OOA+m-SIA & $6.21 \pm 0.67$ & $0.219 \pm 0.045$ & 0.47 \\
\hline OOA+BBOA+HOA+m-SIA & $5.79 \pm 0.73$ & $0.231 \pm 0.046$ & 0.49 \\
\hline OOA+BBOA+m-SIA & $5.91 \pm 0.70$ & $0.229 \pm 0.046$ & 0.49 \\
\hline \multicolumn{4}{|c|}{} \\
\hline OA & Zurich 2013 & $0.398 \pm 0.064$ & 0.55 \\
\hline OA+m-SIA & $6.46 \pm 0.66$ & $0.205 \pm 0.016$ & 0.81 \\
\hline OOA & $6.11 \pm 0.35$ & $0.436 \pm 0.070$ & 0.51 \\
\hline OOA+m-SIA & $8.14 \pm 0.45$ & $0.226 \pm 0.020$ & 0.78 \\
\hline OOA+BBOA+HOA+m-SIA & $6.84 \pm 0.32$ & $0.211 \pm 0.015$ & 0.82 \\
\hline OOA+BBOA+m-SIA & $6.58 \pm 0.30$ & $0.216 \pm 0.017$ & 0.81 \\
\hline
\end{tabular}




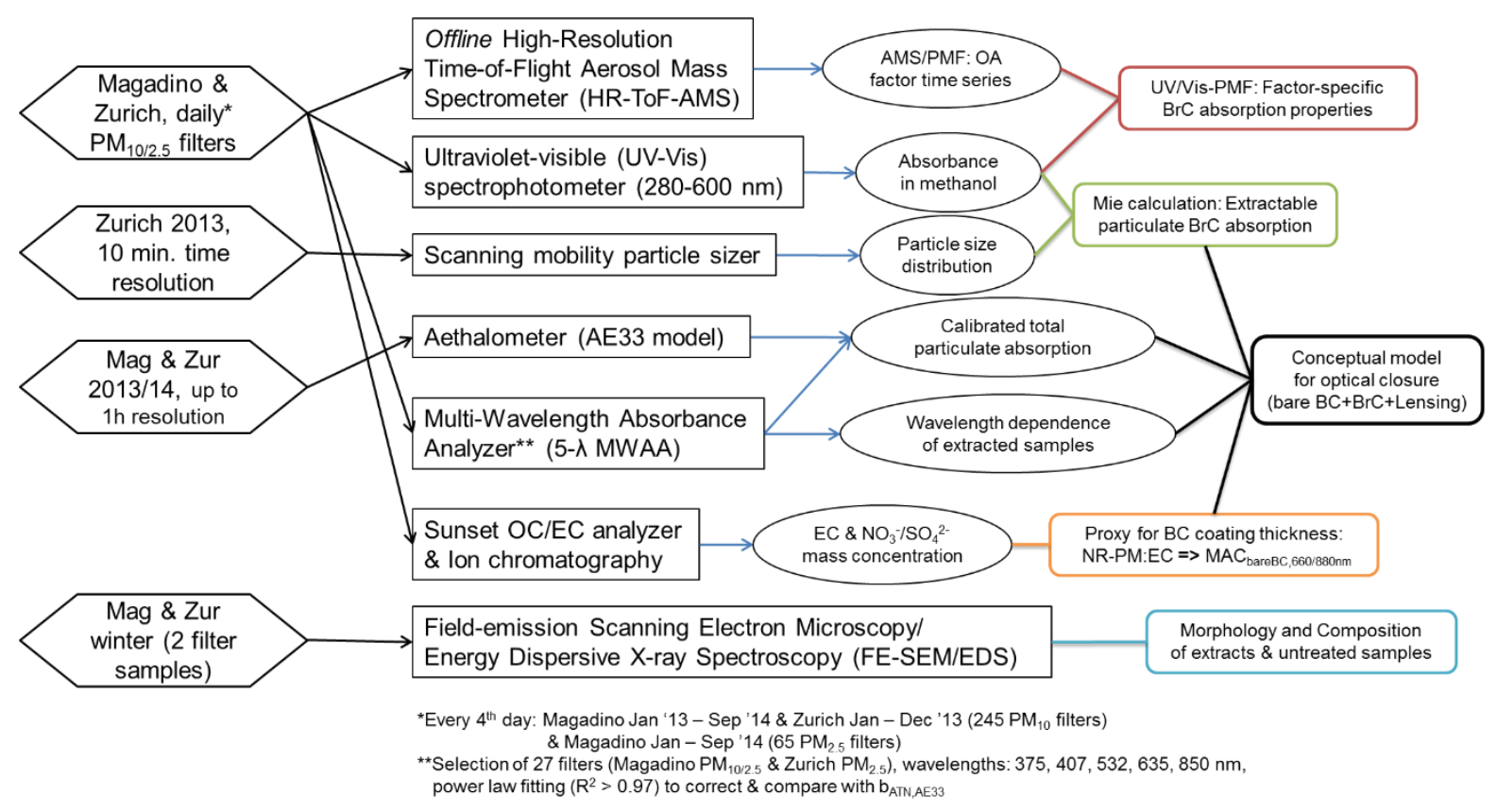

Figure S1. Flowsheet of how to obtain the various (mass) absorption coefficients, EC mass concentration and other aerosol properties from the raw filter-based data delivered by different types of instruments and methods. These were then used to obtain multi-wavelength optical closure for the different absorbing species.
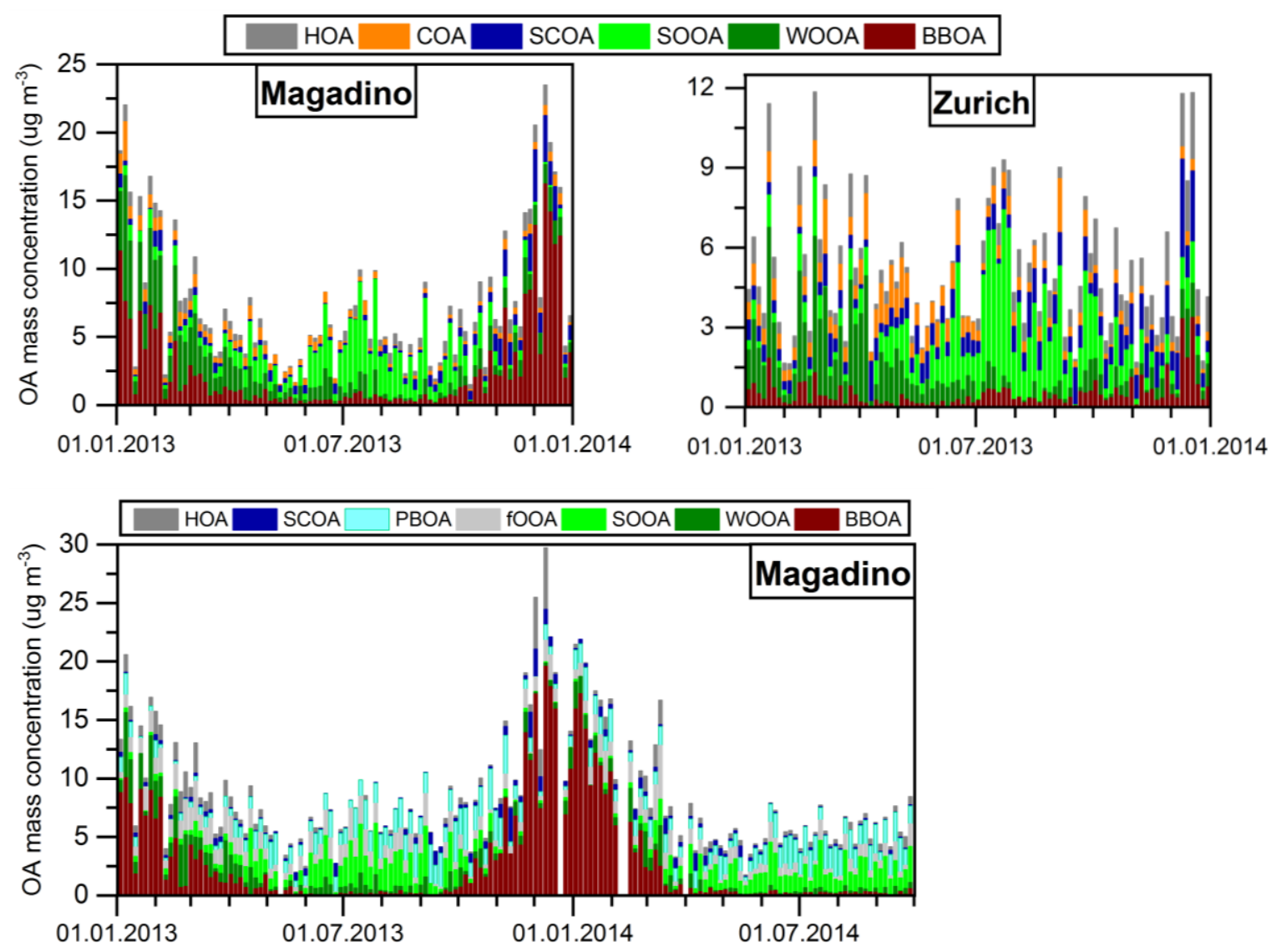

Figure S2. Time series of offline $\mathrm{PM}_{10}$ total OA (by applying source-specific recoveries) AMS/PMF factors (Sect. S2). Upper panels: Solution 2 (Daellenbach et al., 2017); Bottom panel: Solution 1 (Vlachou et al., 2018), used as constraints in UV/Vis-PMF (Sect. 2.2). Note the non-negligible WOOA mass in spring/summer and the important contribution of BBOA in Magadino winter and in Zurich during November-December 2013. 

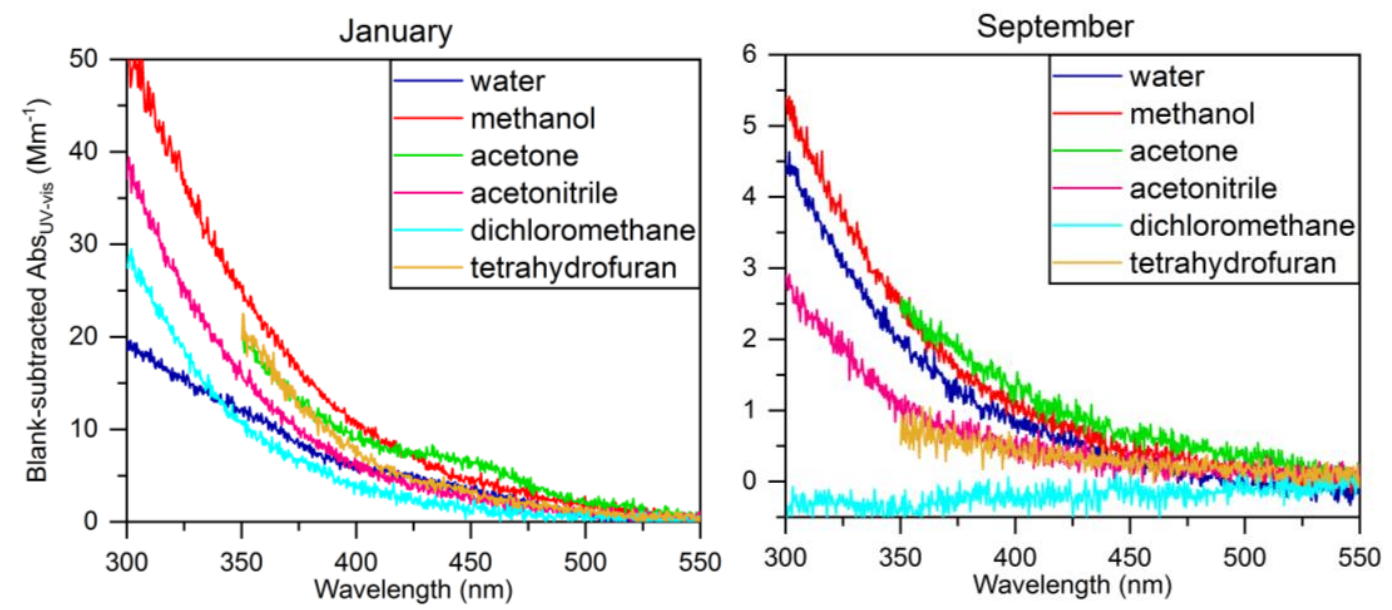

Figure S3. Calculated absorbance for a winter and summer filter extracted in different solvents. Acetone and acetonitrile have a larger dipole moment than methanol, no hydrogen bonds and can dissolve charged species. An absorption hump around $470 \mathrm{~nm}$ was observed at longer wavelengths for water and acetone. Acetone, which is less polar than methanol, provided comparable results but had the disadvantage of a cut-off wavelength of $330 \mathrm{~nm}$, whereas the absorbance in water was significantly lower than in methanol for the Magadino winter sample. The absorbance in dichloromethane (the least polar of the tested solvents) was negligible for the summer sample. Methanol was selected in this study for optical closure purposes considering the obtained absorbance in the full wavelength range, as well as the extraction efficiency of BBOA-dominated samples in methanol (Fig. S6).

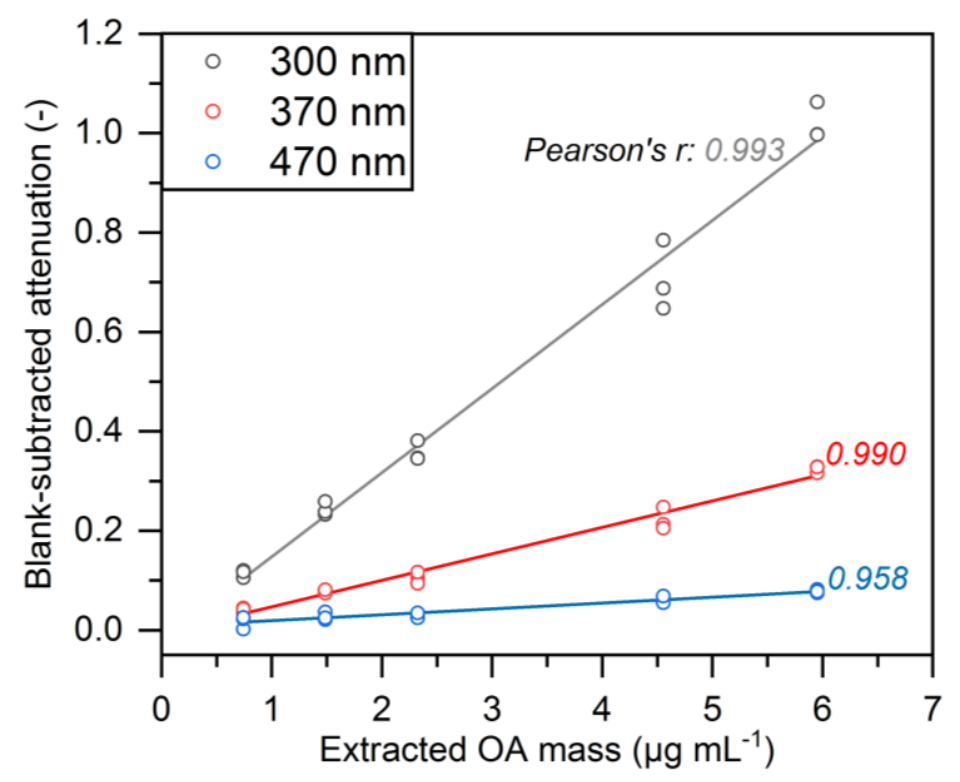

Figure S4. Linearity of $A T N_{\text {sol }}^{10}$ obtained at three different wavelengths for a Magadino $2014 \mathrm{PM}_{2.5}$ filter sample extracted in methanol three times at five different concentrations, covering the full range of our solvent extraction (x-axis) and UV-vis measurement (y-axis) conditions. 


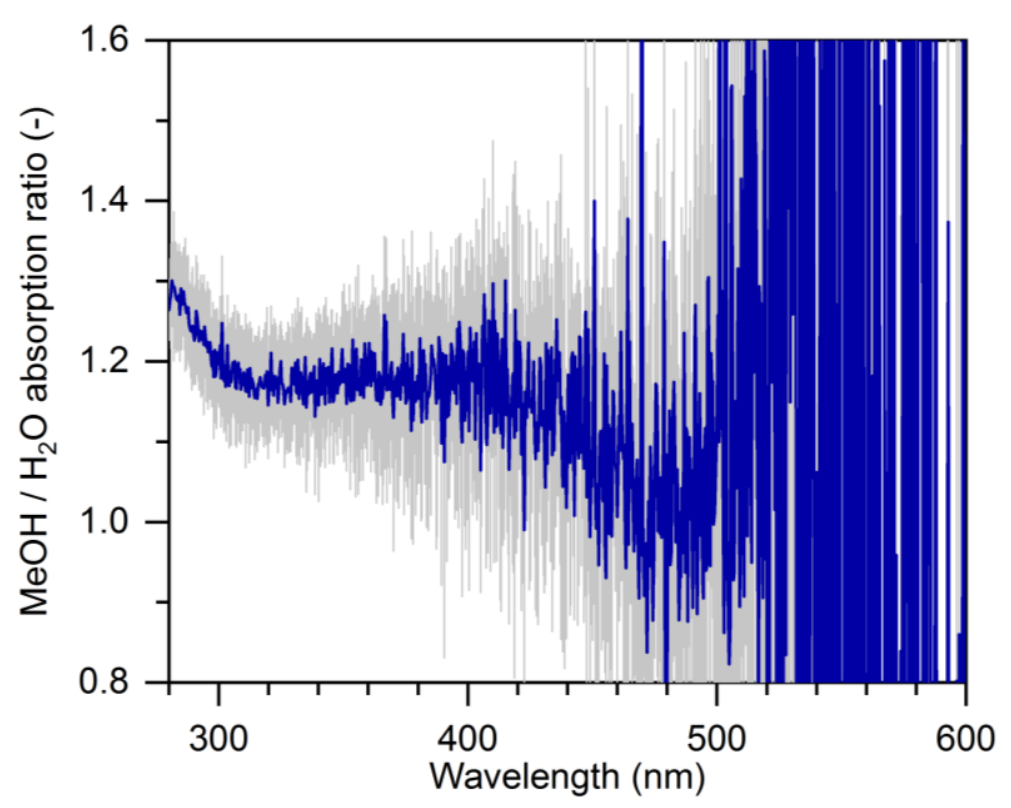

Figure S5. Assessment of the water/methanol solvent effect on UV-vis measurements, performed by comparing the attenuation spectra for ambient PM extracted in water and then diluted in methanol or in water in a 10:90 ratio. The blue curve and gray shading indicate the average and standard deviation, respectively, from 5 Magadino $2014 \mathrm{PM}_{2.5}$ samples. The ratio at 280-470 nm was higher than that calculated in previous study (Phillips and Smith, 2017), whereas above $470 \mathrm{~nm}$ we considered a ratio of 1.0 because the signal was noisy. The water-insoluble (but methanol-soluble) BrC absorbance used in UV/Vis-PMF, $\boldsymbol{A}_{\mathbf{M e O H o n l y}}=\boldsymbol{A}_{\mathbf{M e O H}}-\boldsymbol{A}_{\boldsymbol{H} \mathbf{2} \boldsymbol{o}}$, was corrected for the solvent effect by multiplying $\boldsymbol{A}_{\boldsymbol{H} \mathbf{} \boldsymbol{O}}$ with the average wavelength-dependent absorption ratio below $470 \mathrm{~nm}$.

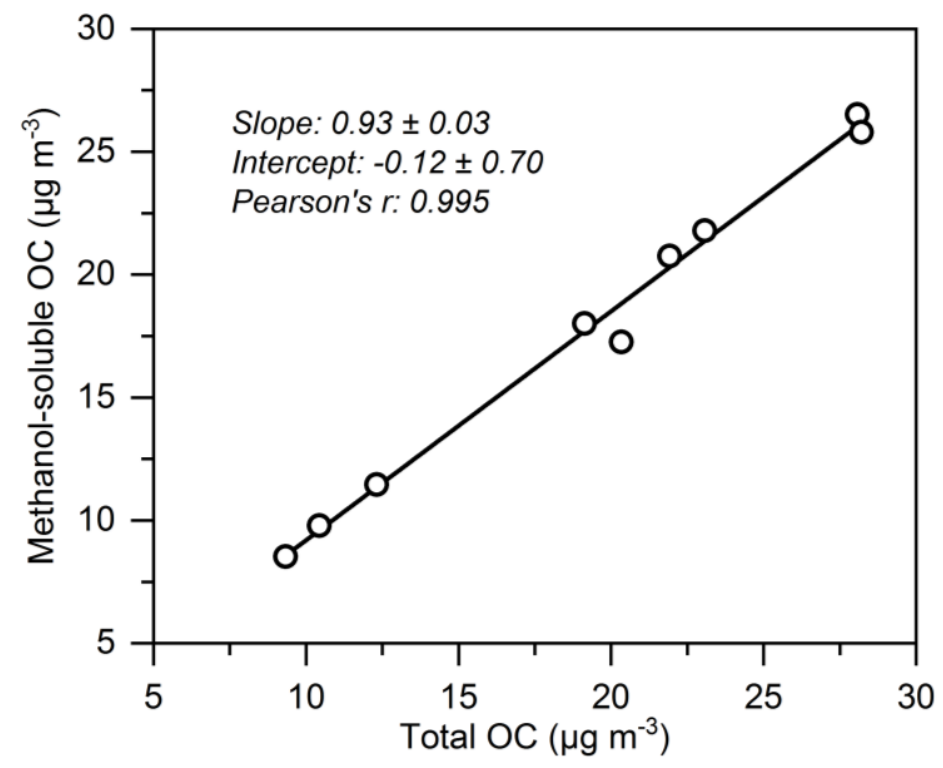

Figure S6. Estimation of BBOA extraction efficiency in methanol, determined by Sunset measurements (EUSAAR2 protocol) of untreated (x-axis) and methanol-extracted $\mathrm{PM}_{10}$ filter samples from Magadino 2014 \& San Vittore (rural) 2013 (November, December \& January) with maximum contribution of BBOA to total OA mass. The $k_{(\text {WINS-)BBOA }}$ were corrected for the (minimum) extraction efficiency $(93 \pm 3 \%)$, by multiplying the total BBOA mass with 0.93 (Text S4). 


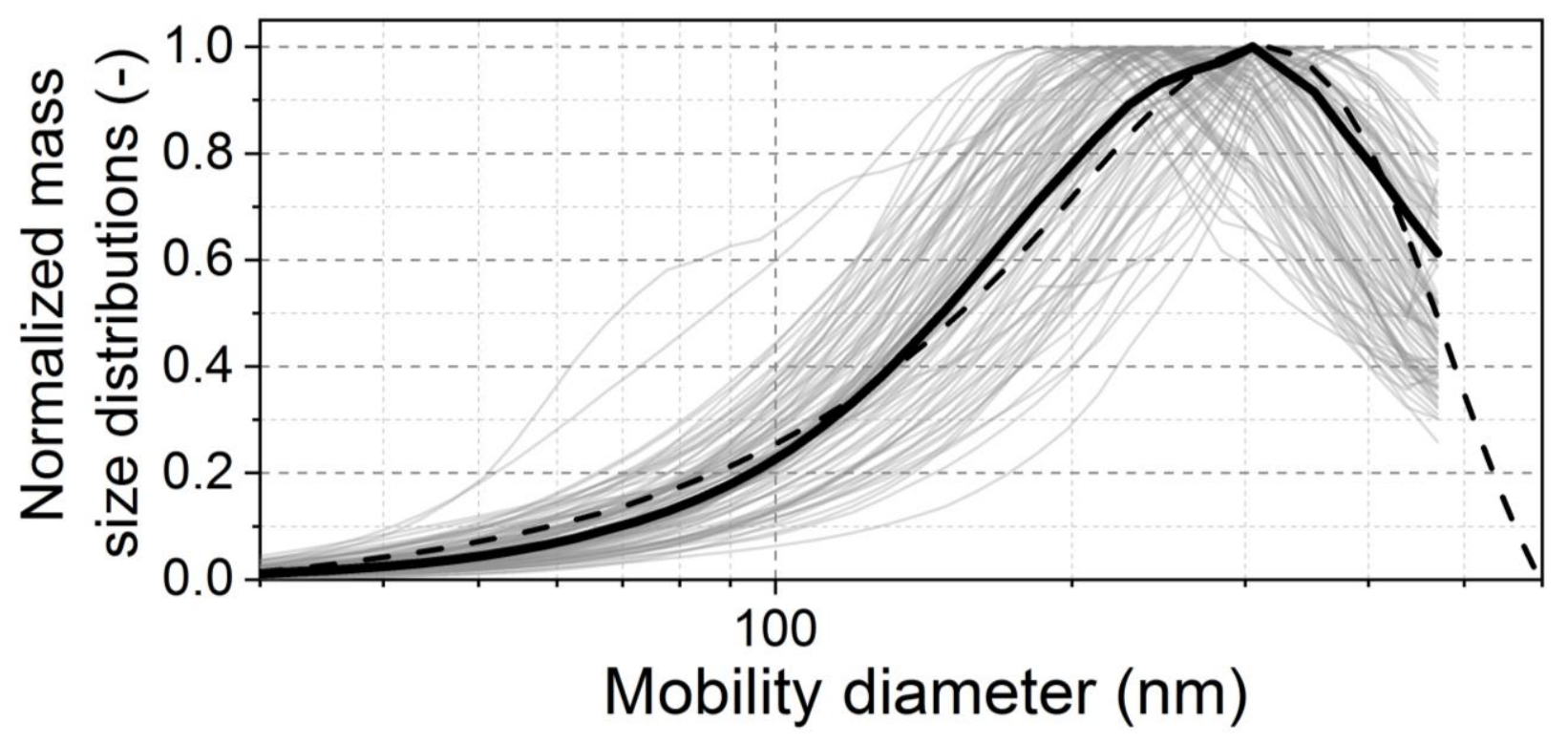

Figure S7. Normalized mass size distributions based on year-long SMPS measurements in Zurich during 2013. Thin grey lines are daily averaged distributions that match the time resolution of the collected offline filters. The thick black line shows the yearly average distribution, extrapolated with the dashed line assuming a log-normal distribution.
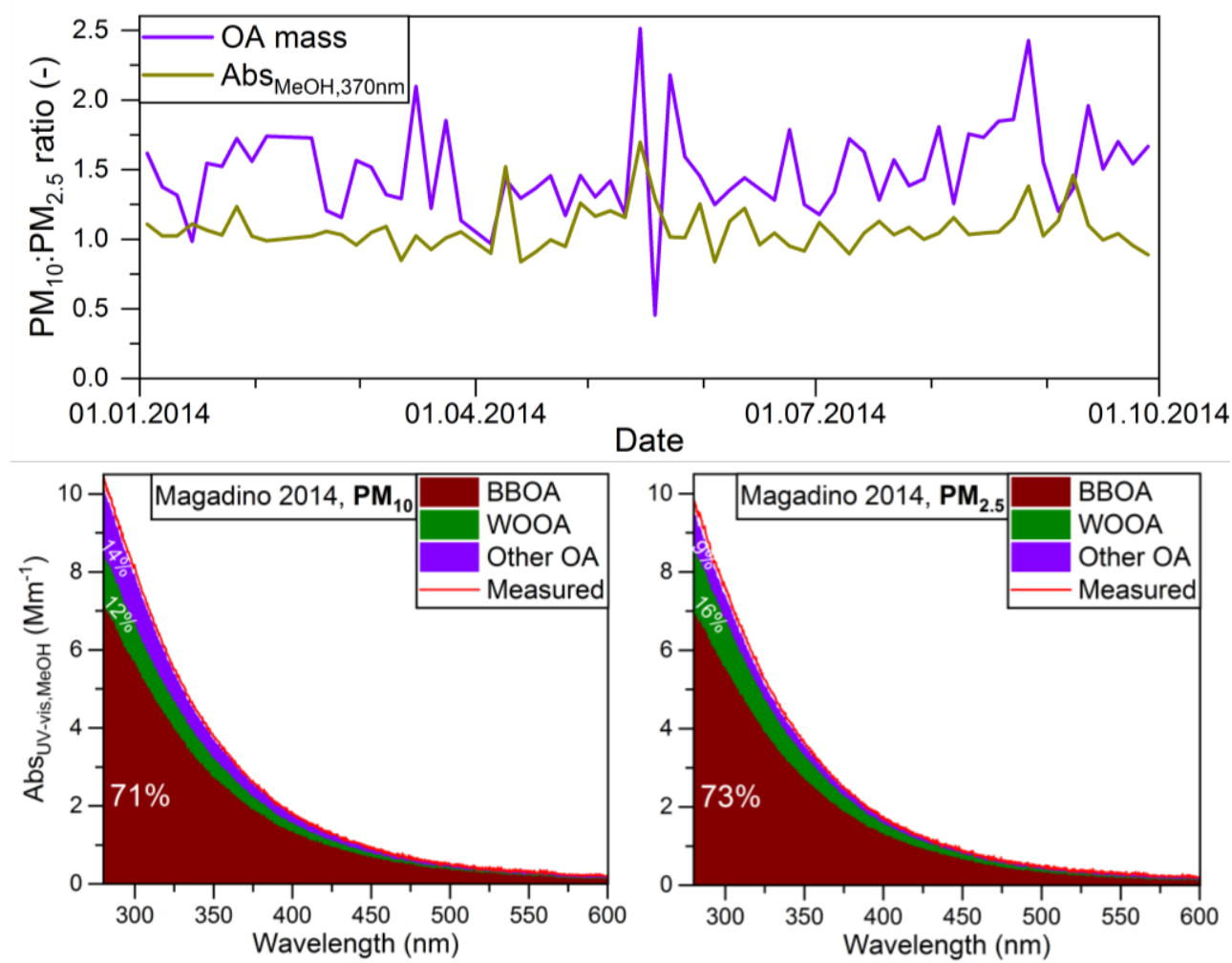

Figure S8. Upper panel: the coarse $\left(\mathrm{PM}_{10}-\mathrm{PM}_{2.5}\right)$ fraction of the aerosol is a negligible contributor to overall absorbance $(\sim 5 \%)$ despite its significant contribution to $\mathrm{PM}_{10}$ mass. Bottom panels: UV/Vis-PMF output for Magadino 2014 (Jan-Sep) where both $\mathrm{PM}_{2.5}$ and $\mathrm{PM}_{10}$ data existed for both AMS/PMF factor mass and UV-vis absorbance, with all 7 factors being used here as model constraints. The model apportioned the absorbance of the two size fractions predominantly and consistently to BBOA and WOOA. The red line shows the measured absorbance (unexplained absorbance $\sim 2-3 \%$ ). 


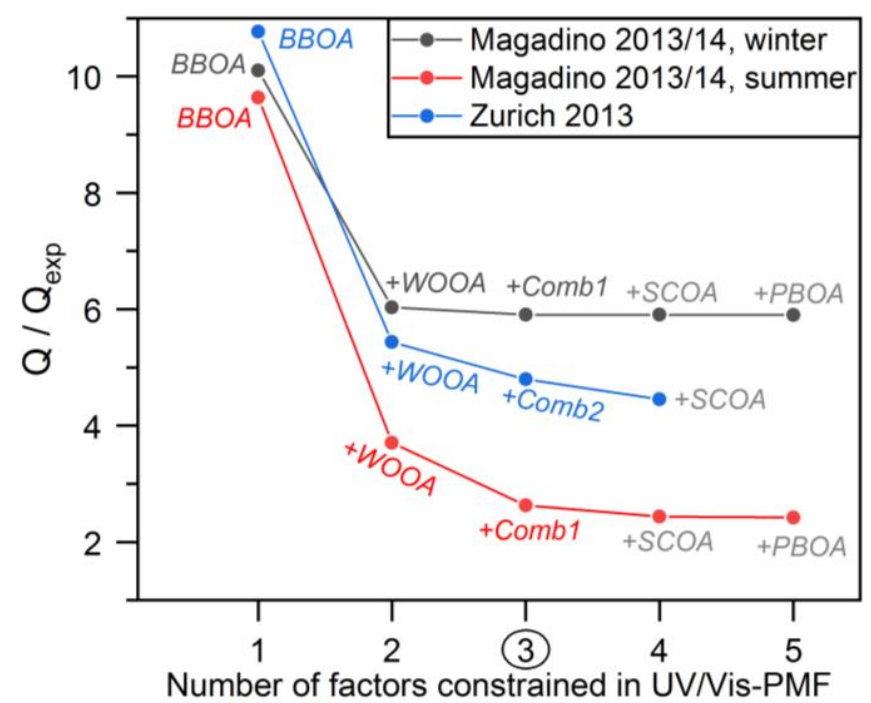

Figure S9. Reduction of scaled residuals $\left(\mathrm{Q} / \mathrm{Q}_{\exp }\right)$ with increasing number of factors constrained in UV/Vis-PMF, clearly showing that BBOA alone did not explain the variability in the measured absorbance. Comb2: SOOA+HOA+COA (AMS/PMF Solution 2); Comb1: SOOA+HOA+AOOA (AMS/PMF Solution 1). BBOA, WOOA and Comb (or "Other OA") were used as model constraints, considering also the correlation analysis shown in Table $\mathrm{S} 1$. The $\mathrm{Q} / \mathrm{Q}_{\exp }$ values for the 3-factor (BBOA, WOOA, Comb) solutions were 5.9, 2.6 and 4.8 for Magadino winter, Magadino summer and Zurich, respectively.
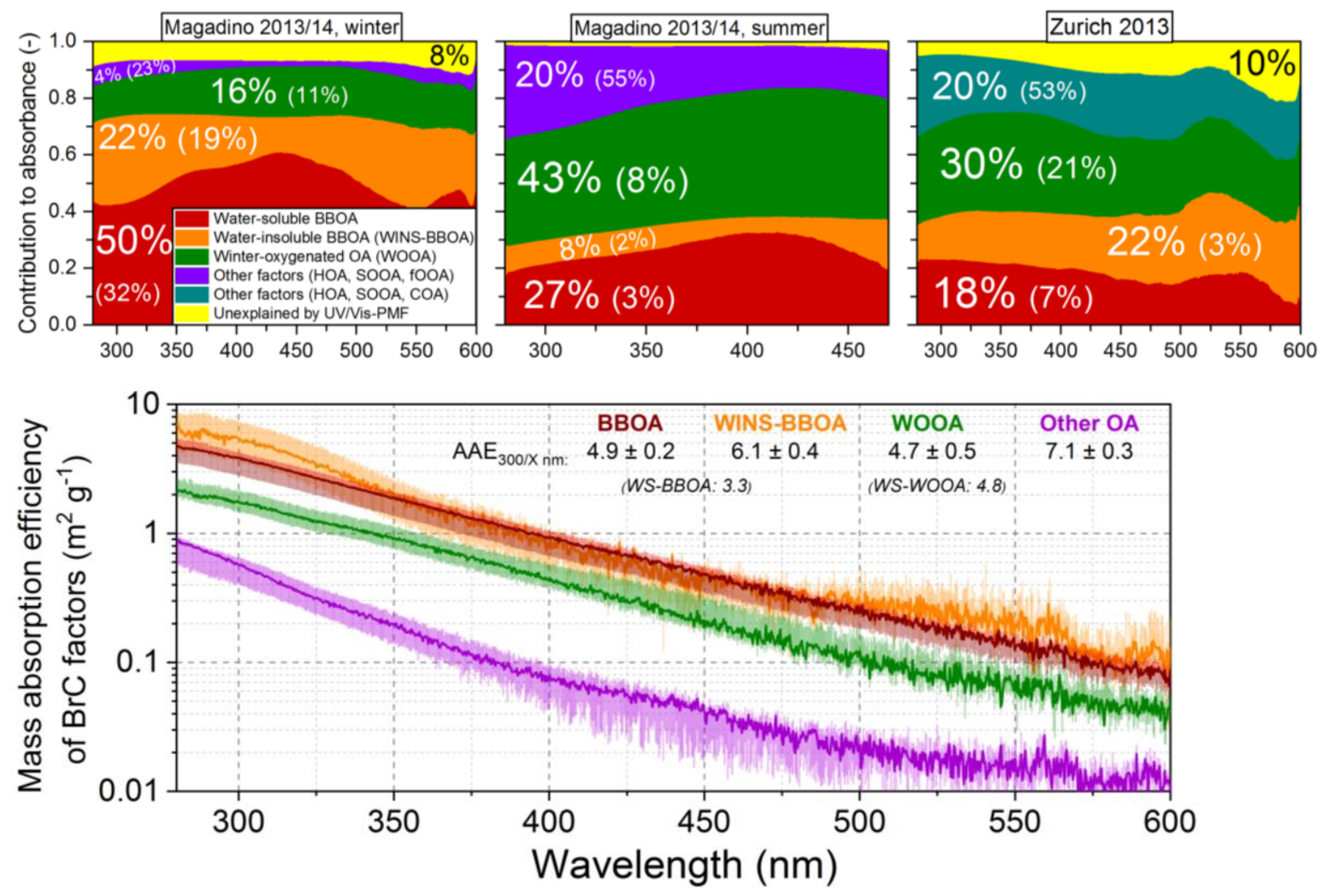

Figure S10. Upper panels: highly spectrally-resolved cumulative relative contributions to absorbance of methanol-extracted $\mathrm{PM}_{10}$ aerosol, apportioned by UV/Vis-PMF analysis (Moschos et al., 2018) to various OA source components resolved by offline aerosol mass spectrometry (Fig. S2), demonstrated for three different model runs. Data were smoothed for demonstration, whereas summer data above $470 \mathrm{~nm}$ are highly uncertain (low absorbance). Labels show the contributions to absorbance averaged across the entire wavelength range, whereas the absorbing (model input) factor contributions to OA mass are shown in parentheses. Bottom panel: absorption properties (AAE, median MAE \& IQR) of the methanol-soluble BrC sources obtained by UV/Vis-PMF, based on sensitivity runs (Text S4; Table S2) including the three case studies shown in the upper panels, and their comparison to the watersoluble (WS) BrC factor AAEs (Moschos et al., 2018). 

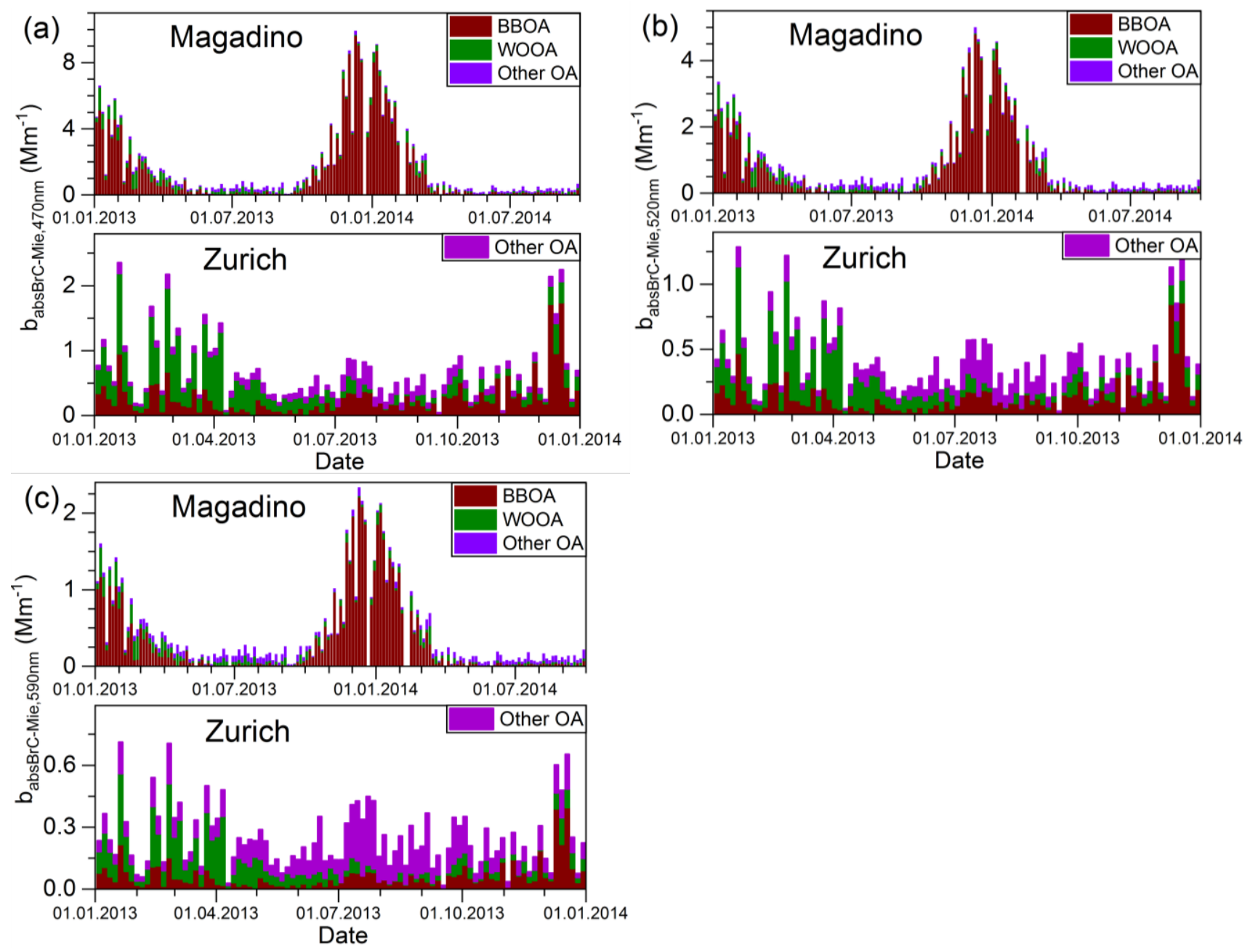

Figure S11. Time series of the average cumulative BBOA, WOOA and Other OA factor contributions to Mie-predicted particulate BrC absorption at $470 \mathrm{~nm}$ (a), $520 \mathrm{~nm}$ (b) and $590 \mathrm{~nm}$ (c) for Magadino and Zurich.
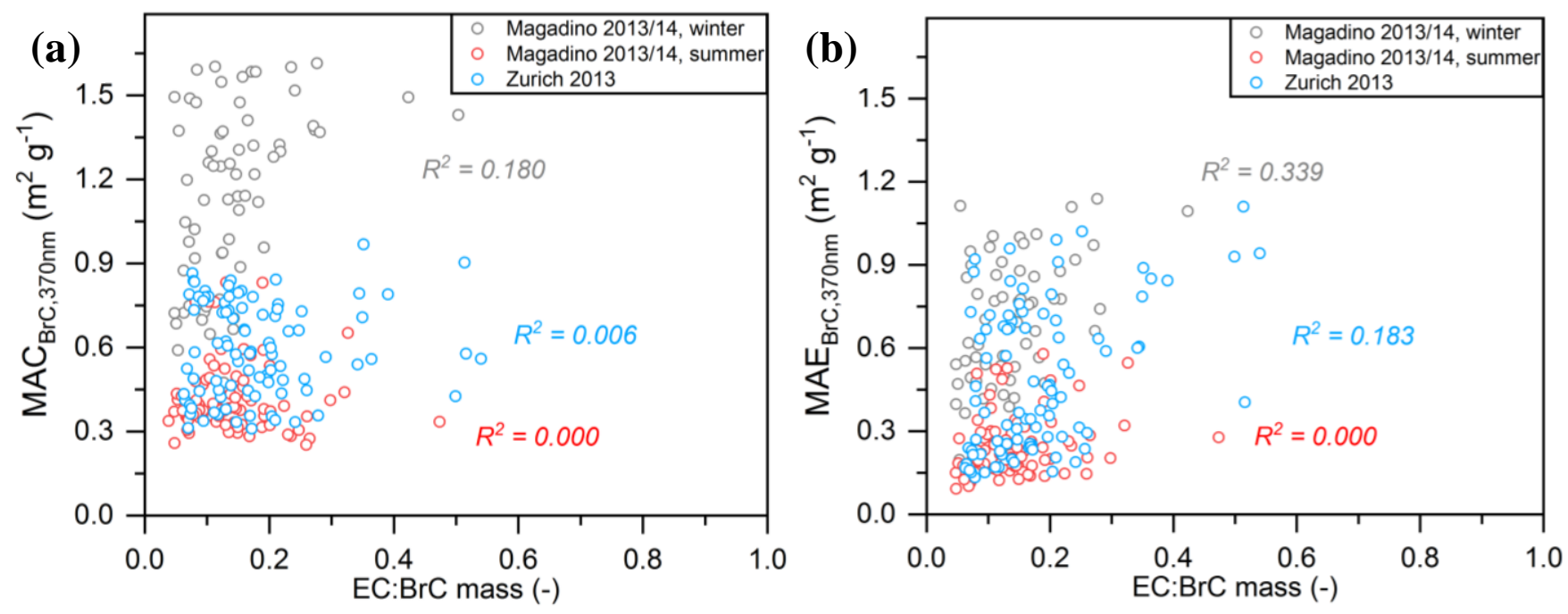

Figure S12. a) Correlation between the average time-resolved $\mathrm{MAC}_{\mathrm{BrC}, 370 \mathrm{~nm}}$ and the EC-to-BrC mass ratio for ambient filter samples from Magadino and Zurich at both seasons. $\mathrm{BrC}$ is considered as the total OA without the contributions of the non-absorbing SCOA and PBOA (i.e., BBOA + WOOA + Other OA). No significant trend is observed, which limits the applicability of the respective parametrization (Saleh et al., 2014) to ambient (mixed-source, aged/processed) samples. b) Same as (a) but with MAE values. 

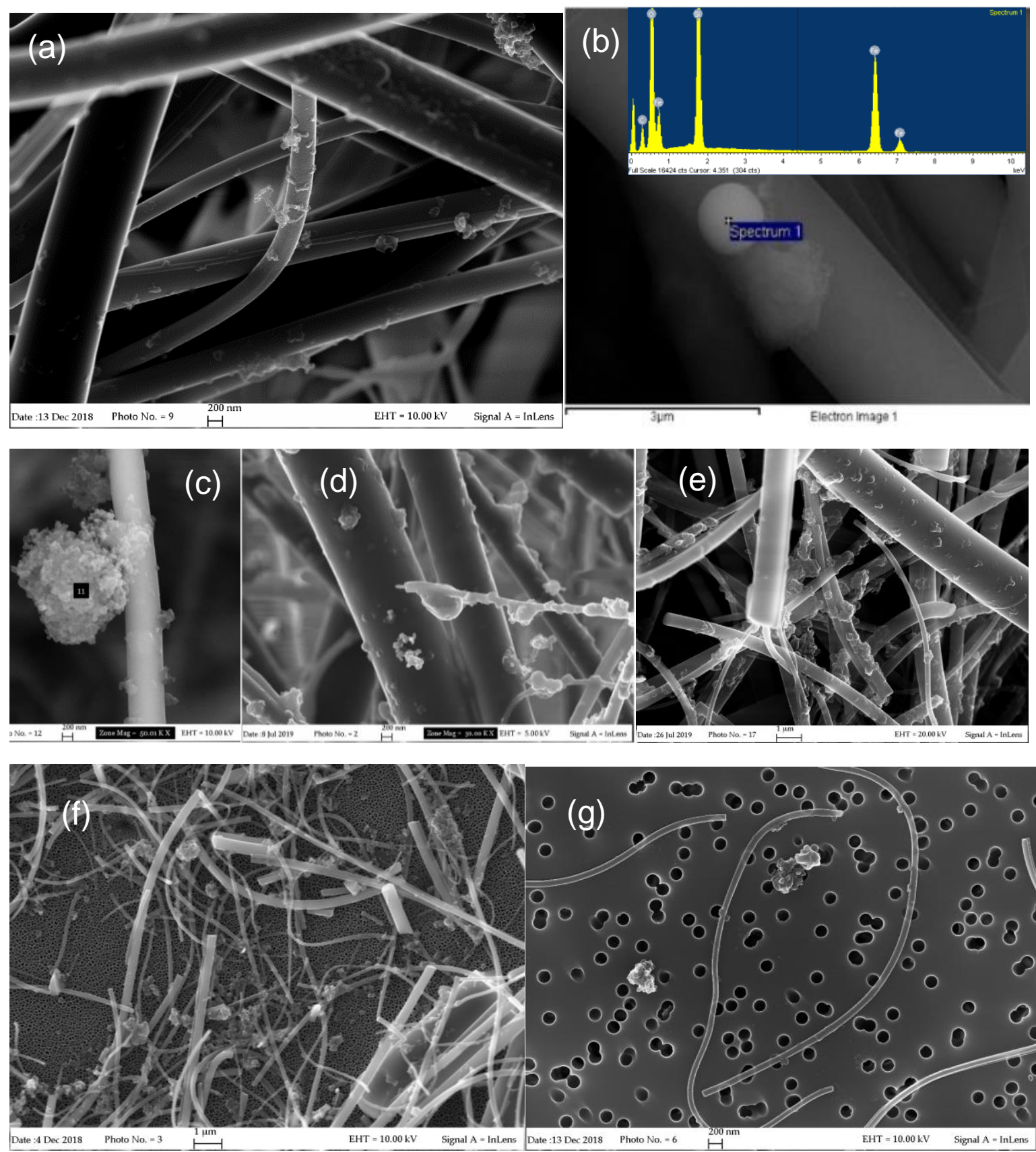

Figure S13. FE-SEM/EDS images of selected untreated filters from Zurich (a \& b) and Magadino (c \& d) winter. Carbon assemblies exhibited scarce adhesion onto the filter fibers and accumulate onto deeper, thinner \& rougher fibers and their intersections (a). Whereas the only spherical particles observed in untreated Zurich samples were inorganics (b), in Magadino both bare -including both lacey \& compact/collapsed- BC (c) and drop-shape bigger pseudo-spherical (d) carbonaceous particles (with traces of K and $\mathrm{S}$, based on EDS analysis) were observed, the latter disappearing after washing with water (e; in-depth magnified view), as observed in many different fields. Note that most of the organic particles/coatings, especially the more volatile ones, have not survived under vacuum and thus are not observable with this technique. f) Alumina filter (Anodisc 25 Whatman, pore size $0.1 \mu \mathrm{m}$ ), on which the supernatant was deposited after sonication of the original quartz microfiber filter in water (Conditions: carbon coated, high vacuum; Voltage: $10 \mathrm{kV}$, signal: SE in-lens), and g) Polycarbonate filter (Isopore membrane track-etched, pore size $0.2 \mu \mathrm{m}$ ), on which the supernatant was filtered after water washing of the original quartz filter, showing that the washing with water seemed to remove (mechanically) a noticeable quantity of carbon nanoparticle aggregates, whereas quartz fibers were also detached. 


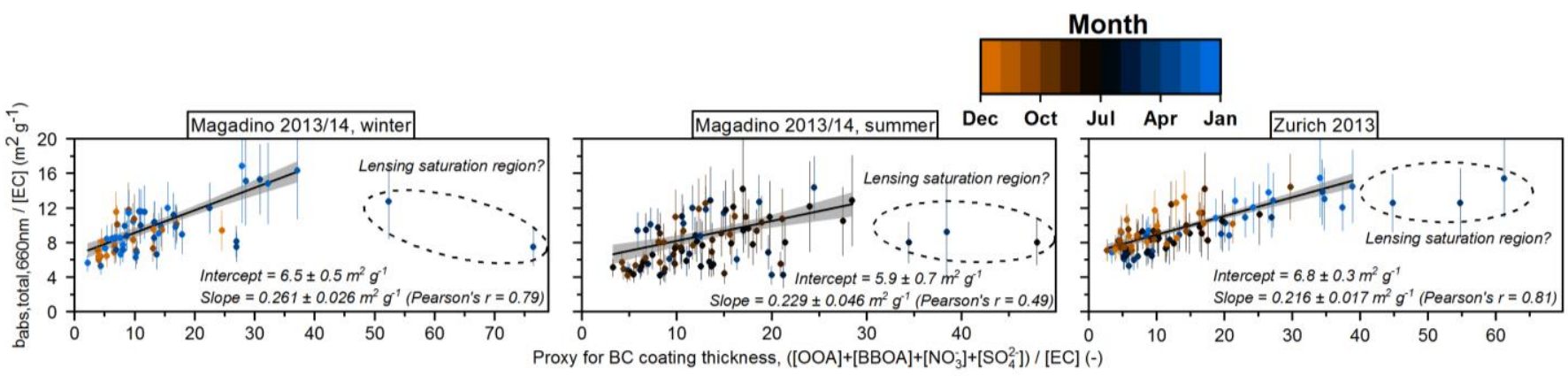

Figure S14. Calculation of $\mathrm{MAC}_{\mathrm{bareBC}}$ as the intercept of uncertainty-weighted linear fits (95\% confidence interval for the slope shown) of $\mathrm{MAC}_{\mathrm{BC}, 660 \mathrm{~nm}} \mathrm{vs}$ the selected proxy for $\mathrm{BC}$ coating thickness, upon obtaining no significant evidence of a source-dependent $\mathrm{MAC}_{\mathrm{BC}}$ (Fig. 4b).

\section{REFERENCES}

Corbin, J. C., Czech, H., Massabò, D., de Mongeot, F. B., Jakobi, G., Liu, F., Lobo, P., Mennucci, C., Mensah, A. A., Orasche, J., Pieber, S. M., Prévôt, A. S. H., Stengel, B., Tay, L. L., Zanatta, M., Zimmermann, R., El Haddad, I., and Gysel, M.: Infraredabsorbing carbonaceous tar can dominate light absorption by marine-engine exhaust, npj Clim. Atmos. Sci., 2, 10.1038/s41612019-0069-5, 2019.

Daellenbach, K. R., Stefenelli, G., Bozzetti, C., Vlachou, A., Fermo, P., Gonzalez, R., Piazzalunga, A., Colombi, C., Canonaco, F., Hueglin, C., Kasper-Giebl, A., Jaffrezo, J.-L., Bianchi, F., Slowik, J. G., Baltensperger, U., El-Haddad, I., and Prévôt, A. S. H.: Long-term chemical analysis and organic aerosol source apportionment at nine sites in central Europe: Source identification and uncertainty assessment, Atmos. Chem. Phys., 17, 13265-13282, 10.5194/acp-17-13265-2017, 2017.

Hecobian, A., Zhang, X., Zheng, M., Frank, N., Edgerton, E. S., and Weber, R. J.: Water-soluble organic aerosol material and the light-absorption characteristics of aqueous extracts measured over the southeastern United States, Atmos. Chem. Phys., 10, 59655977, 10.5194/acp-10-5965-2010, 2010.

Laborde, M., Crippa, M., Tritscher, T., Jurányi, Z., Decarlo, P. F., Temime-Roussel, B., Marchand, N., Eckhardt, S., Stohl, A., Baltensperger, U., Prévôt, A. S. H., Weingartner, E., and Gysel, M.: Black carbon physical properties and mixing state in the European megacity Paris, Atmos. Chem. Phys., 13, 5831-5856, https://doi.org/10.5194/acp-13-5831-2013, 2013.

Massabò, D., Bernardoni, V., Bove, M. C., Brunengo, A., Cuccia, E., Piazzalunga, A., Prati, P., Valli, G., and Vecchi, R.: A multiwavelength optical set-up for the characterization of carbonaceous particulate matter, J. Aerosol Sci., 60, 34-46, 10.1016/j.jaerosci.2013.02.006, 2013.

Moschos, V., Kumar, N. K., Daellenbach, K. R., Baltensperger, U., Prévôt, A. S. H., and El Haddad, I.: Source apportionment of brown carbon absorption by coupling ultraviolet-visible spectroscopy with aerosol mass spectrometry, Environ. Sci. Technol. Lett., 5, 302-308, 10.1021/acs.estlett.8b00118, 2018.

Phillips, S. M., and Smith, G. D.: Spectroscopic comparison of water- and methanol-soluble brown carbon particulate matter, Aerosol Sci. Technol., 51, 1113-1121, 10.1080/02786826.2017.1334109, 2017.

Saleh, R., Robinson, E. S., Tkacik, D. S., Ahern, A. T., Liu, S., Aiken, A. C., Sullivan, R. C., Presto, A. A., Dubey, M. K., Yokelson, R. J., Donahue, N. M., and Robinson, A. L.: Brownness of organics in aerosols from biomass burning linked to their black carbon content, Nat. Geosci., 7, 647-650, 10.1038/ngeo2220, 2014.

Vlachou, A., Daellenbach, K. R., Bozzetti, C., Chazeau, B., Salazar, G. A., Szidat, S., Jaffrezo, J.-L., Hueglin, C., Baltensperger, U., Haddad, I. E., and Prévôt, A. S. H.: Advanced source apportionment of carbonaceous aerosols by coupling offline AMS and radiocarbon size-segregated measurements over a nearly 2-year period, Atmos. Chem. Phys., 18, 6187-6206, 10.5194/acp-18-6187$2018,2018$.

Wolf, R., El-Haddad, I., Slowik, J. G., Dällenbach, K., Bruns, E., Vasilescu, J., Baltensperger, U., and Prévôt, A. S. H.: Contribution of bacteria-like particles to $\mathrm{PM}_{2.5}$ aerosol in urban and rural environments, Atmos. Environ., 160, 97-106, 10.1016/j.atmosenv.2017.04.001, 2017. 